\title{
Numerical Simulation of Heat Transfer in an Enclosure with Time-Periodic Heat Generation Using Finite-Difference Method
}

\author{
Igor Miroshnichenko $^{1(\bowtie)}$ and Mikhail Sheremet ${ }^{2}$ \\ ${ }^{1}$ Regional Scientific and Educational Mathematical Centre, \\ Tomsk State University, 634050 Tomsk, Russia \\ miroshnichenko@mail.tsu.ru \\ 2 Laboratory on Convective Heat and Mass Transfer, \\ Tomsk State University, 634050 Tomsk, Russia \\ sheremet@math.tsu.ru
}

\begin{abstract}
This paper reports a numerical investigation of highly coupled system of partial differential equations, simulating the fluid flow and heat transfer in a large-scale enclosure with time-periodic heat generation. The bottom wall of the enclosure is insulated, and heat exchange with the environment is modeled at other external boundaries. The heater with time-periodic heat generation is located at the bottom of the enclosure. The internal surfaces of both the heater and walls are assumed to be gray. Air is the working fluid and the Rayleigh number is $10^{9}$. To solve the governing equations with dimensionless vorticity stream function - temperature variables, the finite difference method has been used. The developed model has been validated through a comparison with data of other authors. The effect of surface emissivity and periodic heat generation on Nusselt numbers and both stream function and temperature distributions has been investigated. The results showed that the influence of the thermal radiation on total thermal transmission increases with surface emissivity of walls and heater surfaces. The present numerical method can be applied in several engineering problems, such as designing passive cooling systems and the simulation of heat transfer in building constructions.
\end{abstract}

Keywords: Finite-difference method $\cdot$ Heat transfer $\cdot$ Stream function

\section{Introduction}

Many industrial processes are associated with convective heat transfer in large-scale enclosures. One significant application from an energy-saving point of view is an effective optimization of energy consumption for heating buildings. The main and relevant topics for construction and design of buildings are to provide both energy efficiency and thermal comforts for inhabitants. The efficient optimization and thorough design of buildings require modern experimental and numerical approaches. In specialized literature one can find a large number of articles relating to convective heat transfer inside a large-scale enclosure due to its applications in buildings [1-3]. 
Several studies on free convection inside enclosures have been focused on attic space, where the free convection mechanism is sensitive. A comprehensive review of thermal transmission in attic-shaped spaces has been presented by Saha and Khan [4]. Their findings have indicated that most works have been performed in the laminar and transition regimes. At the same time, as practice shows, the airflow in the real attic must be fully turbulent $[5,6]$. Therefore, numerical analysis of hydrodynamics and thermal transmission inside attic (or other large areas) should be carried out in this regime. The review of Das et al. [7] has summarized the studies on natural convection in different (non-square) shapes of enclosures and enclosures with wavy and curved walls. A number of works showed that change of both the angle and aspect ratio of the parallelogrammic and triangular cavities had a big effect on the flow fields. The influence of the different parameters such as the Darcy number, Prandtl number, Rayleigh number, volume fraction of the nanoparticles and irreversibility distribution ratios has also been analyzed. These results provide helpful insight into possible strategies to enhance convective heat transfer inside complicated enclosures. Analysis of the effect of a room heater (location, size and power) on heat transfer and air flow is high important problem. Experimental and numerical simulation of turbulent modes of natural convection in a large-scale cavity with a small heat source has been carried out by Zhang et al. [8]. The central plane velocity fields above the plate have been measured using PIV method and temperature measurements have been obtained using thermocouples. The numerical modeling was conducted by Fluent software using various models of turbulence. The analysis of inertia and buoyancy force discussed in this paper helps to better understand the nature of flow inside the enclosure.

It should be noted that radiative heat transfer plays an important role in thermal transmission inside enclosures. As practice shows, radiation can have a big effect both on hydrodynamic characteristics and heat transfer even at relatively low temperature differences [9-12]. A detailed review of works in the field of turbulent free convection without and with radiation in rectangular cavities was conducted by Miroshnichenko and Sheremet [13]. Inclination angle and shape of the cavities, thermal boundary and initial conditions, various radiative properties and heat source location have also been investigated. Sharma et al. [14] have investigated the interaction effects between turbulent thermogravitational convective heat transport and thermal radiation in bottom heated cavities. The aspect ratio in this study varies from 0.5 to 2 and Ra based on cavity width varies from $10^{8}$ to $10^{12}$. They have found a correlation using aspect ratio and Rayleigh number for determining the mean convective Nusselt number. Kogawa et al. [15] have studied the radiation impact on turbulent modes of free convective energy transport inside closed volume. They have analyzed four different radiation models (surface radiation, non-radiation, gas radiation and combined radiation) to understand the radiation influence of the gas and from the borders. Their results have shown that the effect of gas radiation on radiative energy transport is insignificant, while the surface radiation effect is dominant.

To accurately predict convective-radiative heat transfer, various numerical approaches, namely, finite difference and finite volume methods, finite element and lattice Boltzmann methods are used [16-18]. The impact of non-uniform and uniform heating of inclined walls on free convection in an isosceles triangular cavity has been studied by Basak et al. [19] using the finite element method. They have considered two various cases of thermal boundary conditions, viz., non-uniformly and uniformly 
heating of two inclined walls. The main goal of that study was to analyze the flow and temperature fields with detailed analysis of heat transfer estimates for free convection in triangular cavity. Their findings indicated that geometry does not have much effect on flow structure at small Prandtl numbers.

To the best of our knowledge, investigation of the impact of time periodic heat generation on convective flows in an enclosure having internal heating has not received due attention. The aim of this work is to simulate unsteady turbulent energy transport in a large-scale enclosure with a heater using the finite difference method. Analysis of other heat transfer modes (radiation and conduction inside the heater and solid walls) noticeably affects natural convection and essentially complicates the mathematical model. Moreover, we conducted a comprehensive study of the effect of time-periodic heat generation inside the enclosure.

\section{Mathematical and Physical Models}

The geometry of the problem as shown in Fig. 1 is a large-scale enclosure bounded by massive walls of finite conductivity with a local heat-generating element.

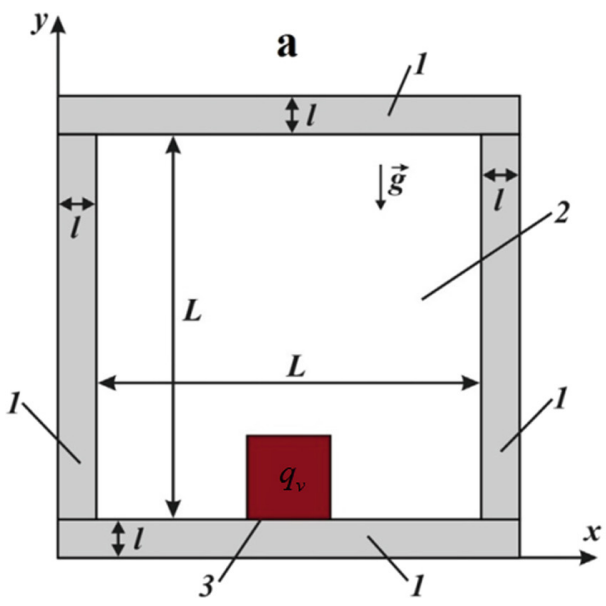

b

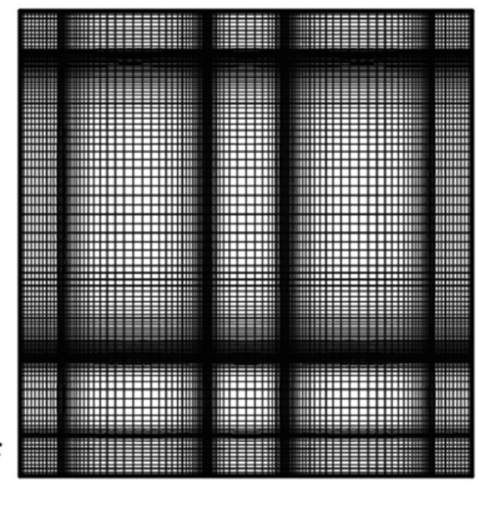

Fig. 1. The domain of interest (a): 1 - solid walls, 2 - air, 3 - heat source of constant temperature, computational domain and grid (b).

The heat-generating element is located on the bottom wall and it has time-periodic heat generation. Time-periodic heat generation was determined as $q=q_{v}(1-\sin (f \cdot t))$. The solid walls of the enclosure are of finite thermal conductivity $\lambda_{w}$ and finite thickness $l$. The external border of the lower wall is adiabatic. No-slip conditions are accepted for cavity walls. The heat exchange with the outside is simulated at external surfaces. All internal walls of the large-scale enclosure are diffuse, opaque and gray emitters. The considered air flow is turbulent in nature. The properties of air are supposed to be permanent except for the density where the Boussinesq approximation is considered. 
Taking into account the above assumptions the Reynolds-averaged Navier-Stokes equations can be written in the following form [20, 21]:

$$
\begin{aligned}
& \frac{\partial G}{\partial t}+\frac{\partial S_{1}}{\partial x_{1}}+\frac{\partial S_{2}}{\partial x_{2}}=R \\
& G=\left(\begin{array}{c}
u_{1} \\
u_{2} \\
T \\
k \\
\varepsilon \\
T \\
T
\end{array}\right) \\
& S_{i}=\left(\begin{array}{c}
u_{i} \\
p \delta_{i 1}-\left(v+v_{t}\right) \sigma_{i 1}+u_{i} u_{1} \\
p \delta_{i 2}-\left(v+v_{t}\right) \sigma_{i 2}+u_{i} u_{2} \\
-\left(\alpha+\alpha_{t}\right) \partial T / \partial x_{i}+u_{i} T \\
-\left(v+v_{t} / \sigma_{k}\right) \partial k / \partial x_{i}+u_{i} k \\
-\left(v+v_{t} / \sigma_{\varepsilon}\right) \partial \varepsilon / \partial x_{i}+u_{i} \varepsilon \\
\alpha_{w} \partial T / \partial x_{i} \\
s \alpha_{h s} \partial T / \partial x_{i}
\end{array}\right), \forall i=1,2 \\
& R=\left(\begin{array}{c}
0 \\
0 \\
g \beta \Delta T \\
0 \\
P_{k}+G_{k}-\varepsilon \\
\left(c_{1 \varepsilon}\left(P_{k}+c_{3 \varepsilon} G_{k}\right)-c_{2 \varepsilon} \varepsilon\right) \frac{\varepsilon}{k} \\
0 \\
q_{v}(1-\sin (f \cdot t))
\end{array}\right) \\
& \sigma_{i j}=\frac{\partial u_{i}}{\partial x_{j}}+\frac{\partial u_{j}}{\partial x_{i}},
\end{aligned}
$$

where $G_{k}$ defines the generation or dissipation of turbulent kinetic energy due to buoyancy, $P_{k}$ describes the production of $k$ and present terms are expressed as

$$
P_{k}=v_{t}\left[2\left(\frac{\partial u_{1}}{\partial x_{1}}\right)^{2}+2\left(\frac{\partial u_{2}}{\partial x_{2}}\right)^{2}+\left(\frac{\partial u_{1}}{\partial x_{2}}+\frac{\partial u_{2}}{\partial x_{1}}\right)^{2}\right], G_{k}=-\frac{g \beta v_{t}}{\operatorname{Pr}_{t}} \frac{\partial T}{\partial x_{2}}
$$


Here $x_{1}, x_{2}$ are the physical coordinates; $T$ is the temperature; $u_{1}, u_{2}$ are the velocity components in the projection on the $x_{1}$ and $x_{2}$ axes, respectively; $\varepsilon$ is the dissipation rate of the kinetic energy of turbulence; $\alpha_{w}$ is the coefficient of thermal diffusivity of the solid wall material; $v$ is the coefficient of kinematic viscosity; $v_{t}$ is the coefficient of turbulent viscosity; $g$ is the gravitational acceleration; $k$ is the kinetic energy of turbulence; $\alpha_{t}$ is the coefficient of turbulent thermal diffusivity; $\beta$ is the temperature coefficient of volume expansion; $t$ is the time; $L$ is the characteristic size of the cavity (Fig. 1). The Kolmogorov-Prandtl formula $v_{t}=c_{\mu} k^{2} / \varepsilon$ was used to calculate turbulent viscosity, $q_{v}$ is the volume density of heat flux.

The governing Eqs. (1)-(5) can be written in a slightly different form, which eliminates the need to search for a pressure field. The form of equations, where the variables are the vorticity $(\omega)$ and stream function $(\psi)$, allows us to reduce the number of differential equations, thereby lead to a decrease in the time needed for calculations. In the present paper, a numerical study of the convective-radiative thermal transmission has been carried out in dimensionless variables. The scales of temperature, velocity, distance, time, kinetic energy of turbulence, dissipation rate of kinetic energy of turbulence, stream function and vorticity are chosen as $\Delta T=T_{h s}-T^{e}, \sqrt{g \beta \Delta T L}, L$, $\sqrt{g \beta \Delta T / L}, g \beta \Delta T L, \sqrt{g^{3} \beta^{3}(\Delta T)^{3} L}, \sqrt{g \beta \Delta T L^{3}}, \sqrt{L / g \beta \Delta T}$, respectively.

With the aim of detailed study of the both energy and momentum transport near the solid walls, a non-uniform grid has been introduced using a special algebraic coordinate transformation $[20,21]$ :

$$
\begin{aligned}
& \xi=a+\frac{b-a}{2}\left\{1+\operatorname{tg}\left[\frac{\pi \kappa}{b-a}\left(X-\frac{a+b}{2}\right)\right] / \operatorname{tg}\left[\frac{\pi}{2} \kappa\right]\right\}, \\
& \eta=a+\frac{b-a}{2}\left\{1+\operatorname{tg}\left[\frac{\pi \kappa}{b-a}\left(Y-\frac{a+b}{2}\right)\right] / \operatorname{tg}\left[\frac{\pi}{2} \kappa\right]\right\} .
\end{aligned}
$$

where $\kappa$ is a compaction parameter and $a, b$ are the geometrical characteristics.

Derivatives of the first and second orders in spatial coordinates are:

$$
\begin{gathered}
\frac{\partial \xi}{\partial X}=\frac{\pi \kappa}{2 \cdot \operatorname{tg}\left\{\frac{\pi \kappa}{2}\right\} \cos ^{2}\left\{\frac{\pi \kappa}{2}(2 X-1)\right\}}, \frac{\partial \eta}{\partial Y}=\frac{\pi \kappa}{2 \cdot \operatorname{tg}\left\{\frac{\pi \kappa}{2}\right\} \cos ^{2}\left\{\frac{\pi \kappa}{2}(2 Y-1)\right\}}, \\
\frac{\partial^{2} \xi}{\partial X^{2}}=\frac{(\pi \kappa)^{2}}{\operatorname{tg}\left\{\frac{\pi \kappa}{2}\right\}} \frac{\sin \left\{\frac{\pi \kappa}{2}(2 X-1)\right\}}{\cos ^{3}\left\{\frac{\pi \kappa}{2}(2 X-1)\right\}}, \frac{\partial^{2} \eta}{\partial Y^{2}}=\frac{(\pi \kappa)^{2}}{\operatorname{tg}\left\{\frac{\pi \kappa}{2}\right\}} \frac{\sin \left\{\frac{\pi \kappa}{2}(2 Y-1)\right\}}{\cos ^{3}\left\{\frac{\pi \kappa}{2}(2 Y-1)\right\}} .
\end{gathered}
$$

The air flow and heat transfer were determined by subsequent characteristics: the Prandtl number $(\mathrm{Pr})$, the Ostrogradsky number $(\mathrm{Os})$ and the Rayleigh number $(\mathrm{Ra})$. These parameters are:

$$
\operatorname{Pr}=\frac{v}{\alpha_{\text {air }}}, O s=\frac{q_{v} L^{2}}{\lambda_{h s} \Delta T}, R a=\frac{g \beta \Delta T L^{3}}{v \alpha_{\text {air }}}
$$


It should be noted that the volumetric heat generation from the heater is described by the Ostrogradsky number. Taking into account the algebraic coordinate transformation noted above boundary and initial conditions are considered in the following form:

At $\tau=0$

$$
\operatorname{Psi}(\xi, \eta, 0)=\Omega(\xi, \eta, 0)=K(\xi, \eta, 0)=E(\xi, \eta, 0)=0, \Theta(\xi, \eta, 0)=0.5
$$

At $\tau>0$

at the boundary $\eta=0: \frac{\partial \Theta}{\partial \eta}=0$;

at the boundaries $\xi=0$ and $\xi=1+2 l / L: \frac{\partial \xi}{\partial X} \frac{\partial \Theta}{\partial \xi}=B i \cdot \Theta$ (the heat exchange with an external environment is simulated);

at the boundary $\eta=1+2 l / L: \frac{\partial \eta}{\partial Y} \frac{\partial \Theta}{\partial \eta}=B i \cdot \Theta$ (the heat exchange with an outside is simulated);

at the heater surface: $\frac{\partial \Theta_{h s}}{\partial \bar{n}}=\frac{\lambda_{\text {air }}}{\lambda_{h s}} \frac{\partial \Theta_{\text {air }}}{\partial \bar{n}}-N_{\text {rad }} Q_{\text {rad }}$;

at the internal surfaces of the walls-air boundary, parallel to the axis $O \xi$ :

$$
\Psi=0, \frac{\partial \Psi}{\partial \eta}=0, \Theta_{w}=\Theta_{\text {air }}, \lambda_{w, \text { air }} \frac{\partial \eta}{\partial Y} \frac{\partial \Theta_{w}}{\partial \eta}=\frac{\partial \eta}{\partial Y} \frac{\partial \Theta_{\text {air }}}{\partial \eta}-N_{\text {rad }} Q_{\text {rad }}
$$

at the internal surfaces of the walls-air boundary, parallel to the axis $O \eta$ :

$$
\Psi=0, \frac{\partial \Psi}{\partial \xi}=0, \Theta_{w}=\Theta_{\text {air }}, \lambda_{w, \text { air }} \frac{\partial \xi}{\partial X} \frac{\partial \Theta_{w}}{\partial \xi}=\frac{\partial \xi}{\partial X} \frac{\partial \Theta_{\text {air }}}{\partial \xi}-N_{\text {rad }} Q_{\text {rad }} .
$$

The boundary conditions for the turbulent characteristics were presented in detail previously in [21]. It is necessary to understand the impact of radiative mechanism of energy transfer on the hydrodynamics and thermal transmission in the analyzed domain. The non-dimensional net radiative thermal flux $Q_{\text {rad }}$ is defined as [21]:

$$
\begin{gathered}
Q_{r a d, k}=R_{k}-\sum_{i=1}^{N} F_{k-i} R_{i} \\
R_{k}=\left(1-\tilde{\varepsilon}_{k}\right) \sum_{i=1}^{N} F_{k-i} R_{i}+\tilde{\varepsilon}_{k}(1-\zeta)^{4}\left(\Theta_{k}+0.5 \frac{1+\zeta}{1-\zeta}\right)^{4} .
\end{gathered}
$$

Here $B i=h L / \lambda_{w}$ is the Biot number, $\sigma$ is the Stefan-Boltzmann constant, $\lambda_{\text {air }}$ is the air thermal conductivity, $N_{\text {rad }}=\sigma T_{h s}^{4} L /\left[\lambda_{\text {air }}\left(T_{h s}-T^{e}\right)\right]$ is the radiation number, $F_{k-i}$ is the view factor from $k$ th unit to the $i$ th unit of the chamber, $\alpha_{i, j}=\alpha_{i} / \alpha_{j}$ is the thermal diffusivity ratio, $\lambda_{i, j}=\lambda_{i} / \lambda_{j}$ is the thermal conductivity ratio, $\zeta=T^{e} / T_{h s}$ is the 
temperature parameter, $Q_{r a d, k}$ is the net radiative thermal flux (dimensionless), $R_{k}$ is the dimensionless radiosity of the $k$ th unit of a chamber, $\tilde{\varepsilon}$ is the surface emissivity.

To solve the set of governing equations the finite-difference method is used. The system (1)-(5) is a combination of elliptic and parabolic equations. So, the secondorder accurate central differences scheme was used to describe the elliptical equation. The successive over relaxation method was used to solve the difference equation. To solve the parabolic equations the locally one-dimensional scheme was adopted. The diffusion terms in parabolic equations were discretized using central differences scheme of the second-order accuracy, whereas accurate upwind difference scheme was applied to discretize the convective terms. First-order scheme was employed for the transient term. The resulting systems of linear equations were worked out by tridiagonal matrix algorithm (Thomas method).

Next, we consider in detail the solution of elliptic equations for the stream function. Taking into account the mesh transformation, a spatio-temporal uniform grid was constructed:

$$
\xi_{i}=i h_{\xi}, \quad \eta_{j}=j h_{\eta}, \quad \tau_{n}=n \tau,
$$

here $h_{\xi}, h_{\eta}$ - space steps; $\tau$ - time step; $i=\overline{0, N_{\xi}} ; j=\overline{0, N_{\eta}} ; n=\overline{0, M}$.

Denote $f\left(\xi_{i}, \eta_{j}, \tau_{n}\right)=f_{i, j}^{n}$, to approximate the derivatives of the first and second orders, the central differences are used:

$$
\begin{array}{ll}
\frac{\partial f}{\partial \xi} \approx \frac{f_{i+1, j}-f_{i-1, j}}{2 h_{\xi}}, & \frac{\partial^{2} f}{\partial \xi^{2}} \approx \frac{f_{i+1, j}-2 f_{i, j}+f_{i-1, j}}{h_{\xi}^{2}}, \\
\frac{\partial f}{\partial \eta} \approx \frac{f_{i, j+1}-f_{i, j-1}}{2 h_{\eta}}, & \frac{\partial^{2} f}{\partial \eta^{2}} \approx \frac{f_{i, j+1}-2 f_{i, j}+f_{i, j-1}}{h_{\eta}^{2}} .
\end{array}
$$

The approximation relation for the time derivative is as follows:

$$
\frac{\partial f}{\partial \tau} \approx \frac{f_{i, j}^{n+1}-f_{i, j}^{n}}{\tau}
$$

Poisson's equation for the stream function taking into account the coordinate transformation is defined as:

$$
\frac{d^{2} \xi}{d X^{2}} \frac{\partial \Psi}{\partial \xi}+\left(\frac{d \xi}{d X}\right)^{2} \frac{\partial^{2} \Psi}{\partial \xi^{2}}+\frac{d^{2} \eta}{d Y^{2}} \frac{\partial \Psi}{\partial \eta}+\left(\frac{d \eta}{d Y}\right)^{2} \frac{\partial^{2} \Psi}{\partial \eta^{2}}=-\Omega
$$

One approach to solving elliptic equations is to use central differences to approximate second-order derivatives. As a result, we obtain the following discrete equation: 


$$
\begin{aligned}
\frac{d^{2} \xi}{d X^{2}} \frac{\Psi_{i+1, j}^{k}-\Psi_{i-1, j}^{k+1}}{2 h_{\xi}} & +\left(\frac{d \xi}{d X}\right)^{2} \frac{\Psi_{i+1, j}^{k}-2 \Psi_{i, j}^{k+1}+\Psi_{i-1, j}^{k+1}}{h_{\xi}^{2}}+\frac{d^{2} \eta}{d Y^{2}} \frac{\Psi_{i, j+1}^{k}-\Psi_{i, j-1}^{k+1}}{2 h_{\eta}} \\
& +\left(\frac{d \eta}{d Y}\right)^{2} \frac{\Psi_{i, j+1}^{k}-2 \Psi_{i, j}^{k+1}+\Psi_{i, j-1}^{k+1}}{h_{\eta}^{2}}=-\Omega_{i, j}
\end{aligned}
$$

here $k$ is a number of iterations.

Denote

$$
\frac{d \xi}{d X}=a, \frac{d^{2} \xi}{d X^{2}}=b, \frac{d \eta}{d Y}=c, \frac{d^{2} \eta}{d Y^{2}}=d .
$$

Further, the resulting system of linear algebraic equations is solved by successive over relaxation method:

$$
\left\{\begin{aligned}
\Psi_{i, j}^{k+1}= & \frac{h_{\eta}^{2} h_{\xi} b\left(\Psi_{i+1, j}^{k}-\Psi_{i-1, j}^{k+1}\right)}{4\left(c^{2} h_{\xi}^{2}+a^{2} h_{\eta}^{2}\right)}+\frac{h_{\eta}^{2}\left(\Psi_{i+1, j}^{k}+\Psi_{i-1, j}^{k+1}\right)}{2\left(c^{2} h_{\xi}^{2}+a^{2} h_{\eta}^{2}\right)}+\frac{h_{\xi}^{2} h_{\eta} d\left(\Psi_{i, j+1}^{k}-\Psi_{i, j-1}^{k+1}\right)}{4\left(c^{2} h_{\xi}^{2}+a^{2} h_{\eta}^{2}\right)} \\
& +\frac{h_{\xi}^{2}\left(\Psi_{i, j+1}^{k}+\Psi_{i, j-1}^{k+1}\right)}{2\left(c^{2} h_{\xi}^{2}+a^{2} h_{\eta}^{2}\right)}+\frac{h_{\xi}^{2} h_{\eta}^{2} \Omega_{i, j}}{2\left(c^{2} h_{\xi}^{2}+a^{2} h_{\eta}^{2}\right)}, \\
& \Psi_{i, j}^{k+1}=\Psi_{i, j}^{k}+\varpi\left(\widehat{\Psi}_{i, j}^{k+1}-\Psi_{i, j}^{k}\right) .
\end{aligned}\right.
$$

The relaxation parameter $\varpi$ was chosen experimentally from the results of many numerical experiments. A thorough criterion for stream function variable is used to receive converged solutions at each time step. The convergence condition $\left|\Psi_{i j}^{k+1}-\Psi_{i j}^{k}\right|<10^{-6}$ must be satisfied by variable $\Psi_{i j}^{k}$ at any grid point $(i, j)$, here $k$ is a given iteration parameter. This difference scheme is unconditionally stable and has an approximation order $O\left(h_{\xi}^{2}+h_{\eta}^{2}\right)$.

Table 1. Variation of the average Nusselt numbers

\begin{tabular}{l|l|l|l|l}
\hline $\mathrm{Ra}$ & {$[22]$} & {$[23]$} & {$[24]$} & Present data \\
\hline $10^{7}$ & 16.79 & 16.523 & - & 17.13 \\
\hline $10^{8}$ & 30.506 & 30.225 & 28.78 & 33.06 \\
\hline $10^{9}$ & 57.35 & - & 62.0 & 60.54 \\
\hline
\end{tabular}

In order to verify the numerical algorithm, the developed computational code has been validated successfully using various numerical results. In the case of natural convection inside the differentially heated enclosure, the developed computational code 
has been validated successfully using the numerical results (variation of the average Nusselt number) of Dixit and Babu [22], Zhuo and Zhong [23] and Le Quere [24] (Table 1).

The numerical simulation has been carried out on coarser and finer meshes, in order to check the grid independence. These computational tests with various grids allowed choosing the suitable grid size selection without compromising both accuracy and CPU time. The mesh sensitivity investigation was examined for $\tilde{\varepsilon}=0, \mathrm{Ra}=10^{9}$. Three different meshes of $60 \times 60,120 \times 120$ and $156 \times 156$ were applied to verify the grid independence. The fluid flow rate, average convective Nusselt number at the heater surface and average heater temperature are shown in Table 2. The obtained results have shown that the $120 \times 120$ grid provided a good accuracy. For example, the maximum difference in terms of the average convective Nusselt number between case of $120 \times 120$ points and case of $156 \times 156$ points is less than $5.6 \%$. In this connection, the non-uniform $120 \times 120$ grid has been selected for analysis.

Table 2. Grid independence study for $\mathrm{Ra}=10^{9}, \tilde{\varepsilon}=0, f=0$.

\begin{tabular}{l|l|l|l}
\hline Grid size & $N u_{\text {conv }}$ & $|\psi|_{\max }$ & $\Theta_{h s}$ \\
\hline $60 \times 60$ & 48.78 & 0.031 & 0.764 \\
\hline $120 \times 120$ & 61.54 & 0.028 & 0.783 \\
\hline $156 \times 156$ & 64.974 & 0.027 & 0.786 \\
\hline
\end{tabular}

\section{Results and Discussion}

Numerical simulation is reported for the following values of key parameters: $\operatorname{Pr}=0.7$, $\mathrm{Ra}=10^{9}, h / L=0.1, \zeta=0.82, O s=1, N_{\text {rad }}=245.36,0 \leq \tilde{\varepsilon} \leq 1$. The main attention was paid to the impact of both surface emissivity and time-periodic heat generation on distributions of both integral parameters (average radiative and convective Nusselt numbers at the heater surface) and local parameters (streamlines and isotherms). The geometry (Fig. 1) is selected with an eye to simulate thermal transmission and air motion within the room with a heater. The present results are received using one Intel Core i7 processor of $3.30 \mathrm{GHz}$ with $16 \mathrm{~GB}$ memory RAM.

Figure 2 shows the distribution of isolines of the stream function and temperature as a function of the surface emissivity $(\tilde{\varepsilon}=0.3-a, \tilde{\varepsilon}=0.9-b)$ at $f=0.001 \pi$. Two lowintensity symmetric convective cells determining clockwise (right) and counterclockwise (left) circulations are formed inside the large-scale enclosure. The formation of the present vortex structures is caused by cooling of the analyzed area due to the heat exchange with the environment (since the ambient temperature is lower than the initial temperature inside the enclosure), as well as the influence of the heater which located on the bottom wall of the enclosure. The presence of an upward flow of warm air in the central part of the cavity reflects the formation of a thermal plume. The bottom solid wall is a zone least affected by the environment, this is due to heat dissipation from the heat source. With an increase in the surface emissivity, the intensity of convective flow decreases, which is confirmed by the characteristic decrease in the maximum value of the stream function in the core of the convective cell $|\Psi|_{\max }^{\tilde{\varepsilon}=0.9}=0.0255<|\Psi|_{\max }^{\tilde{\varepsilon}=0.3}=0.0269$. Just to clarify 
again, thermal transmission is caused by both the effect of time-periodic heat generation from the heater and the cooling of the enclosure owing to the heat exchange with an environment. The radiation significantly affects the distribution of temperature inside the cavity. In general, an increment of surface emissivity allows increasing the mean temperature inside the air-filled enclosure.

$a$
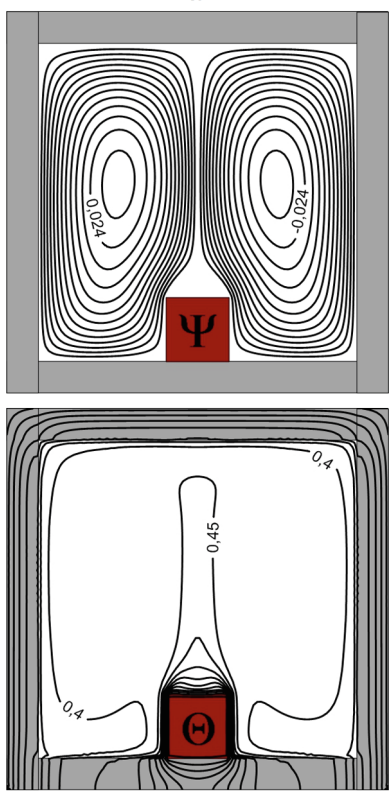

$b$
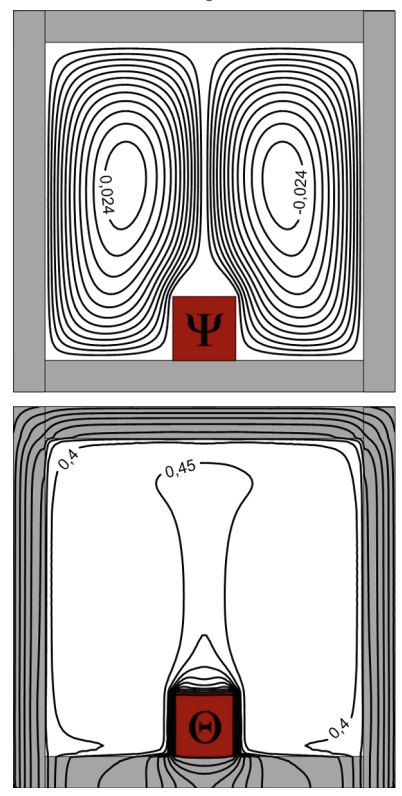

Fig. 2. Isotherms $\Theta$ and streamlines $\Psi$ for different values of surface emissivity at $f=0.001 \pi$ : $\tilde{\varepsilon}=0.3-a, \tilde{\varepsilon}=0.9-b$

The impact of the surface emissivity of heater and walls surfaces, as well as dimensionless time on the average convective and radiative Nusselt numbers (at the surface of the heater) has been analyzed numerically in Fig. 3. It should be noted that if the boundary conditions are not isothermal (for example, volumetric heat generation from the heater) then achieving the steady state conditions is extremely difficult. Timeperiodic heat generation was determined as $q=q_{v}(1-\sin (f \tau))$. When surface emissivity values increase, the temperature gradient at the heater surface is reduced and natural convection is also weakened. So, with an increase in $\tilde{\varepsilon}$, a characteristic decrease in the intensity of convective heat transfer has been observed (at $\tau=5000$, the average convective Nusselt number decreases by $8.1 \%$ when changing of $\tilde{\varepsilon}$ from 0.3 to 0.9 ), while a growth of the average radiative Nusselt number with increasing surface emissivity values has been observed (at $\tau=5000$, a change of $\tilde{\varepsilon}$ from 0.3 to 0.9 leads to an increase in the average radiative Nusselt number up to 3.47 times). Although profiles are time-periodic, the peaks and valleys are increasing with time. This is due to the fact that heat conduction equation inside the heater has an internal heat generation term. 
More detailed influence of the values of surface emissivity on the temperature profiles at the middle cross-Sect. $10=0.6$ is depicted in Fig. 4. A rise of surface emissivity of internal surfaces affects a slight decreasing temperature directly within the heater. This fact also leads to a growth of the mean temperature within the large-scale enclosure. It should be noted that the increase in surface emissivity is manifested in a noticeable decrease in temperature inside the heater.
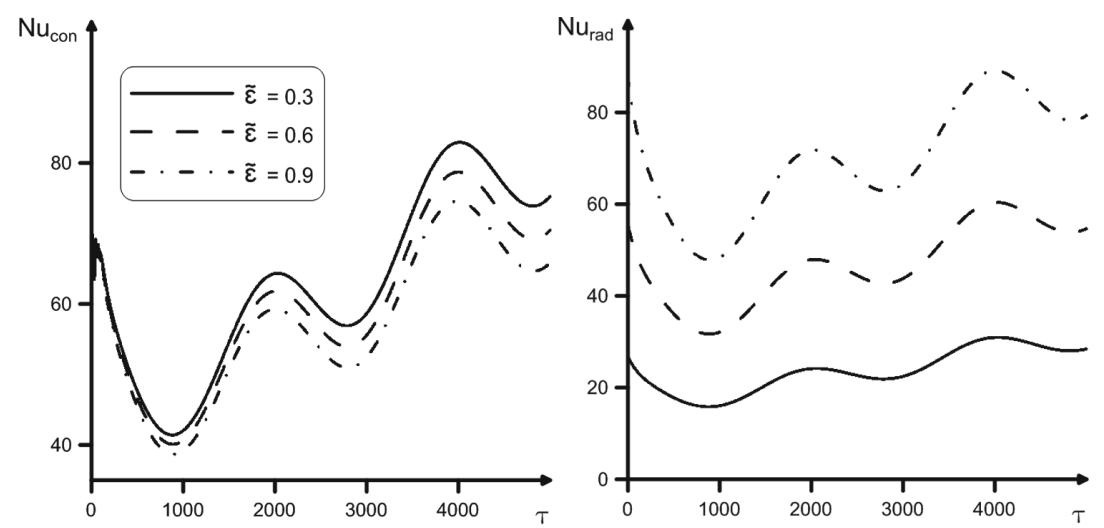

Fig. 3. Variation of the average convective $\left(N u_{c o n}\right)$ and radiative $\left(N u_{r a d}\right)$ Nusselt numbers at the heat source surface with the dimensionless time and the surface emissivity $\tilde{\varepsilon}$ at $f=0.001 \pi$.

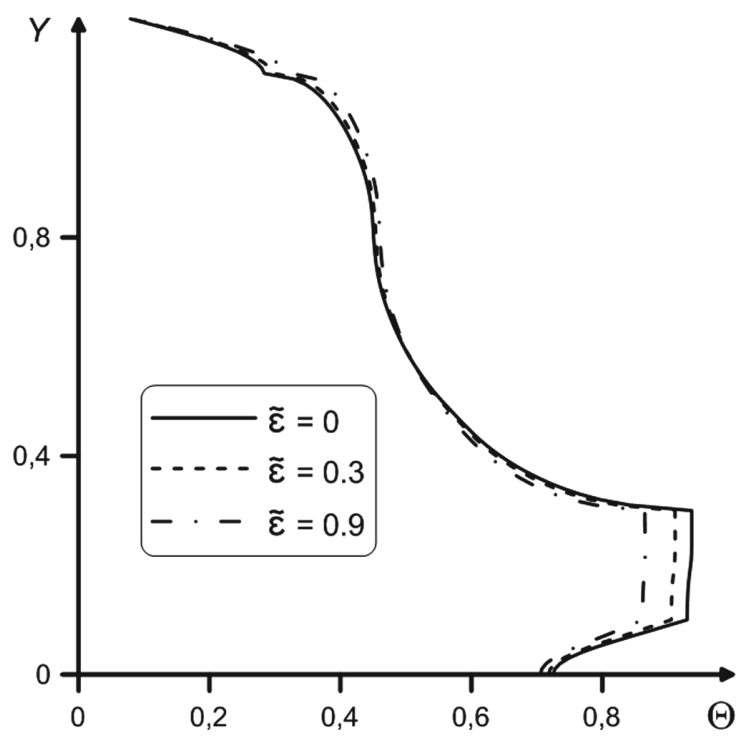

Fig. 4. Temperature profiles at $X=0.6$ for different values of surface emissivity 


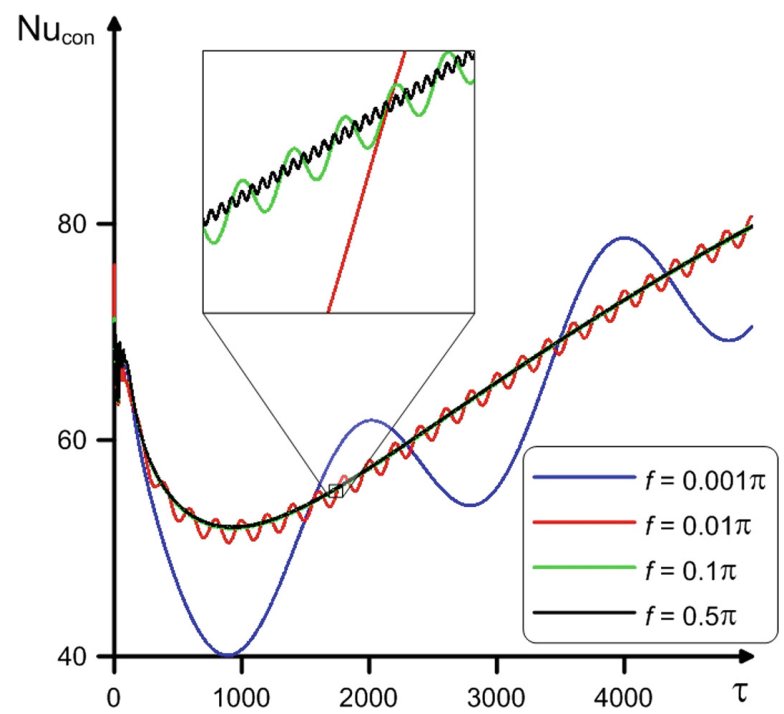

Fig. 5. Dependence of the mean convective Nusselt number at the heater surface vs. parameter $f$ and non-dimensional time at $\tilde{\varepsilon}=0.6$.

In order to study the influence of time-periodic heat generation on energy transport, the convective heat exchange from the heater was estimated by the average convective Nusselt number. Figure 5 presents the average convective Nusselt number for various surface emissivities at the heater-air boundary. The results have indicated that there is no time point which characterizes the stationary distribution of heat transfer coefficients. This is due to time-periodic heat generation inside the local heater. It is worth noting that a decrease in parameter $f$ leads to growth of period of oscillation.

\section{Conclusions}

Numerical simulation of convective-radiative heat transfer in a large-scale enclosure with heat-conducting walls of finite thickness in the presence of local energy source with timeperiodic heat generation has been carried out. To generate the systems of linear equations using vorticity and stream function, the finite difference technique has been employed. The developed computational code has been validated through a comparison with data of other authors. As a result of the research, various distributions of integral and local parameters characterizing the hydrodynamic and thermal transmission in the enclosure have been obtained. A role of the natural convection in the total energy transport decreases with an increase in the surface emissivity of both solid walls and heater. It has been shown that in a large-scale enclosures with one heat-generating element under conditions of convective heat exchange with external environment the heat removal from the heatgeneration element can be increased even with small growth of $\tilde{\varepsilon}$. The surface radiation has a significant effect on the total heat exchange and can reach more than $50 \%$ of the total heat flux, particularly if the heater and wall surfaces have high emissivity. 
Acknowledgements. This work was supported by the Russian Science Foundation (Project No. 19-79-00296).

\section{References}

1. Li, X., Tu, J.: Evaluation of the eddy viscosity turbulence models for the simulation of convection-radiation coupled heat transfer in indoor environment. Energy Build. 184, 8-18 (2019)

2. Rahimi, M., Sabernaeemi, A.: Experimental study of radiation and free convection in an enclosure with a radiant ceiling heating system. Energy Build. 42, 2077-2082 (2010)

3. Muresan, C., Menezo, C., Bennacer, R., Vaillon, R.: Numerical simulation of a vertical solar collector integrated in a building frame: radiation and turbulent natural convection coupling. Heat Transfer Eng. 27, 29-42 (2006)

4. Saha, S.C., Khan, M.M.K.: A review of natural convection and heat transfer in attic-shaped space. Energy Build. 43, 2564-2571 (2011)

5. Ridouane, E., Campo, A., Hasnaoui, M.: Turbulent natural convection in an air-filled isosceles triangular enclosure. Int. J. Heat Fluid Flow 27, 476-489 (2006)

6. Ghasemi, M., Fathabadi, M., Shadaram, A.: Numerical analysis of turbulent natural convection heat transfer inside a triangular-shaped enclosure utilizing computational fluid dynamic code. Int. J. Numer. Methods Heat Fluid Flow 18, 14-23 (2008)

7. Das, D., Roy, M., Basak, T.: Studies on natural convection within enclosures of various (non-square) shapes - a review. Int. J. Heat Mass Transfer 106, 356-406 (2017)

8. Zhang, X., Su, G., Yu, J., Yao, Z., He, F.: PIV measurement and simulation of turbulent thermal free convection over a small heat source in a large enclosed cavity. Build. Environ. 90, 105-113 (2015)

9. Velusamy, K., Sundararajan, T., Seetharamu, K.N.: Interaction effects between surface radiation and turbulent natural convection in square and rectangular enclosures. J. Heat Transfer 123, 1062-1070 (2001)

10. Shati, A.K.A., Blakey, S.G., Beck, S.B.M.: A dimensionless solution to radiation and turbulent natural convection in square and rectangular enclosures. J. Eng. Sci. Technol. 7, 257-279 (2012)

11. Vivek, V., Sharma, A.K., Balaji, C.: Interaction effects between laminar natural convection and surface radiation in tilted square and shallow enclosures. Int. J. Thermal Sci. 60, 70-84 (2012)

12. Martyushev, S.G., Miroshnichenko, I.V., Sheremet, M.A.: Numerical analysis of spatial unsteady regimes of conjugate convective-radiative heat transfer in a closed volume with an energy source. J. Eng. Phys. Thermophys. 87, 124-134 (2014)

13. Miroshnichenko, I.V., Sheremet, M.A.: Turbulent natural convection heat transfer in rectangular enclosures using experimental and numerical approaches: a review. Renew. Sustain. Energy Rev. 82, 40-59 (2018)

14. Sharma, A.K., Velusamy, K., Balaji, C., Venkateshan, S.P.: Conjugate turbulent natural convection with surface radiation in air-filled rectangular enclosures. Heat Mass Transfer 50, 625-639 (2007)

15. Kogawa, T., Okajima, J., Sakurai, A., Komiya, A., Maruyama, S.: Influence of radiation effect on turbulent natural convection in cubic cavity at normal temperature atmospheric gas. Int. J. Heat Mass Transfer 104, 456-466 (2017)

16. Wang, H., Xin, S., Le Quere, P.: Numerical study of natural convection-surface radiation coupling in air-filled square cavities. C.R. Mecanique 334, 48-57 (2006) 
17. Alvarado, R., Xaman, J., Hinojosa, J., Alvarez, G.: Interaction between natural convection and surface thermal radiation in tilted slender cavities. Int. J. Thermal Sciences 47, 355-368 (2008)

18. Nia, M.F., Nassab, S.A.G., Ansari, A.B.: Transient combined natural convection and radiation in a double space cavity with conducting walls. Int. J. Therm. Sci. 128, 94-104 (2018)

19. Basak, T., Roy, S., Krishna Babu, S., Balakrishnan, A.R.: Finite element analysis of natural convection flow in a isosceles triangular enclosure due to uniform and non-uniform heating at the side walls. Int. J. Heat Mass Transf. 51, 4496-4505 (2008)

20. Miroshnichenko, I.V., Sheremet, M.A.: Effect of thermal conductivity and emissivity of solid walls on time-dependent turbulent Conjugate convective-radiative heat transfer. J. Appl. Comput. Mech. 5(2), 207-216 (2019)

21. Miroshnichenko, I.V., Sheremet, M.A.: Numerical simulation of turbulent natural convection combined with surface thermal radiation in a square cavity. Int. J. Numer. Methods Heat Fluid Flow 25, 1600-1618 (2015)

22. Dixit, H.N., Babu, V.: Simulation of high rayleigh number natural convection in a square cavity using the Lattice Boltzmann Method. Int. J. Heat Mass Transf. 49, 727-739 (2006)

23. Zhuo, C., Zhong, C.: LES-based filter-matrix lattice boltzmann model for simulating turbulent natural convection in a square cavity. Int. J. Heat Fluid Flow 42, 10-22 (2013)

24. Le Quéré, P.: Accurate solutions to the square thermally driven cavity at High Rayleigh number. Comput. Fluids 20, 29-41 (1991) 